\title{
On Sobolev-type Inequalities on Morrey Spaces of an Integral Form
}

\author{
Takao Ohno* and Tetsu Shimomura
}

\begin{abstract}
We prove Sobolev-type inequalities for modified Riesz potentials of functions in Morrey spaces of an integral form over non-doubling metric measure spaces. Our results are new even for the doubling metric measure setting. In particular, our results extend the previous results in Morrey spaces of an integral form in the Euclidean case.
\end{abstract}

\section{Introduction}

For $0<\alpha<N$ and a locally integrable function $f$ on $\mathbf{R}^{N}$ the Riesz potential $U_{\alpha} f$ of order $\alpha$ is defined by

$$
U_{\alpha} f(x)=\int_{\mathbf{R}^{N}}|x-y|^{\alpha-N} f(y) d y .
$$

The classical Sobolev inequality says that the Riesz potential $U_{\alpha} f$ of order $\alpha$ with $f \in$ $L^{p}\left(\mathbf{R}^{N}\right)$ belongs to $L^{p^{*}}\left(\mathbf{R}^{N}\right)$ when $1<p<\infty$ and $1 / p^{*}=1 / p-\alpha / N>0$ (see, e.g. 2 , Theorem 3.1.4(b)]). Morrey spaces were introduced by C. B. Morrey 17 in 1938 to study the existence and regularity of partial differential equations. Sobolev's inequality for Morrey spaces was studied by D. R. Adams [1]. We also refer to [4, 12, 14, 18, 22, etc.

In [15], the second author and Mizuta studied a Sobolev-type inequality for $U_{\alpha} f$ for locally integrable functions $f$ on $\mathbf{R}^{N}$ satisfying

$$
\sup _{x \in G}\left(\int_{0}^{d_{G}} r^{\nu-N} \varphi(r)\left(\int_{B(x, r)}|f(y)|^{p} d y\right) \frac{d r}{r}\right)^{1 / p}<\infty,
$$

where $0<\nu \leq N, G$ is a bounded open set in $\mathbf{R}^{N}, d_{G}=\sup \{d(x, y): x, y \in G\}$ and $\varphi$ is positive monotone functions on the interval $(0, \infty)$ satisfying the conditions $(\varphi)$ and (i) in 15$]$.

We denote by $(X, d, \mu)$ a metric measure space, where $X$ is a bounded set, $d$ is a metric on $X$ and $\mu$ is a nonnegative complete Borel regular outer measure on $X$ which is finite in every bounded set. We often write $X$ instead of $(X, d, \mu)$. For $x \in X$ and $r>0$, we denote by $B(x, r)$ the open ball in $X$ centered at $x$ with radius $r$ and $d_{X}=\sup \{d(x, y): x, y \in X\}$.

Received August 8, 2021; Accepted February 15, 2022.

Communicated by Sanghyuk Lee.

2020 Mathematics Subject Classification. Primary: 46E30; Secondary: 42B25.

Key words and phrases. Riesz potential, maximal functions, Sobolev's inequality, Morrey spaces, metric measure space, non-doubling measure.

${ }^{*}$ Corresponding author. 
We assume that $d_{X}<\infty, \mu(\{x\})=0$ for $x \in X$ and $0<\mu(B(x, r))<\infty$ for $x \in X$ and $r>0$ for simplicity. We do not assume that $\mu$ has a so-called doubling condition. Recall that a Radon measure $\mu$ is said to be doubling if there exists a constant $c_{0}>0$ such that $\mu(B(x, 2 r)) \leq c_{0} \mu(B(x, r))$ for all $x \in \operatorname{supp}(\mu)(=X)$ and $r>0$ (see [3] $)$. Otherwise $\mu$ is said to be non-doubling. For examples of non-doubling metric measure spaces we refer to 21,26 . In connection with the $5 r$-covering lemma, the doubling condition had been a key condition in harmonic analysis. However, Nazarov, Treil and Volberg showed that the doubling condition is not necessary by using the modified maximal operator [19, 20]. In this paper, we show that this is the case for the modified Riesz potential operator.

For $\alpha>0$ and $\tau \geq 1$, we define the (modified) Riesz potential of order $\alpha$ for a locally integrable function $f$ on $X$ by

$$
I_{\alpha, \tau} f(x)=\int_{X} \frac{d(x, y)^{\alpha} f(y)}{\mu(B(x, \tau d(x, y)))} d \mu(y)
$$

(e.g., see [6, 16,23,26]). Note here that we can not reduce the number $\tau$ any more (see [25]), which is based on the idea of Stempak [27]. This is equal to $U_{\alpha} f$ when $X=\mathbf{R}^{N}$ and $\mu=d x$. In the doubling metric measure setting, we use $I_{\alpha, 1} f$. For another type of Riesz potentials like

$$
I_{\eta} f(x)=\int_{X} \frac{f(y)}{\mu(B(x, d(x, y)))^{1-\eta}} d \mu(y)
$$

see [5, 10].

To obtain general results, we consider a weight function $\omega(r):(0, \infty) \rightarrow(0, \infty)$ satisfying the following conditions:

$(\omega 0) \omega(\cdot)$ is continuous on $(0, \infty)$;

$(\omega 1) \omega(\cdot)$ is almost increasing on $(0, \infty)$, namely there exists a constant $\widetilde{c}_{1} \geq 1$ such that

$$
\omega\left(r_{1}\right) \leq \widetilde{c}_{1} \omega\left(r_{2}\right) \quad \text { whenever } 0<r_{1}<r_{2}<\infty \text {; }
$$

$(\omega 2)$ there exists a constant $\widetilde{c}_{2}>1$ such that

$$
\widetilde{c}_{2}^{-1} \omega(r) \leq \omega(2 r) \leq \widetilde{c}_{2} \omega(r) \quad \text { whenever } r>0 ;
$$

( $\omega 3)$ there exist constants $\omega_{0}>0$ and $\widetilde{c}_{3} \geq 1$ such that

$$
\widetilde{c}_{3}^{-1} r^{\omega_{0}} \leq \omega(r) \leq \widetilde{c}_{3} \quad \text { for all } 0<r \leq 2 d_{X}
$$

Example 1.1. Let $0<\sigma<\omega_{0}$ and $\beta \in \mathbf{R}$. Then

$$
\omega(r)=r^{\sigma}(\log (e+1 / r))^{\beta}
$$

satisfies $(\omega 0),(\omega 1),(\omega 2)$ and $(\omega 3)$. 
Recall that $f$ is a locally integrable function on $X$ if $f$ is an integrable function on all balls $B$ in $X$. Let $p \geq 1$ and $\theta \geq 1$. In connection with (1.1), given $\omega(r)$ as above, we define the $\mathcal{L}^{p, \omega, \theta}$ norm by

$$
\begin{aligned}
\|f\|_{\mathcal{L}^{p, \omega, \theta}(X)}=\inf \{\lambda>0 ; & \\
& \left.\sup _{x \in X}\left(\int_{0}^{2 d_{X}} \frac{\omega(r)}{\mu(B(x, \theta r))}\left(\int_{B(x, r)}\left(\frac{|f(y)|}{\lambda}\right)^{p} d \mu(y)\right) \frac{d r}{r}\right) \leq 1\right\} .
\end{aligned}
$$

The space of all measurable functions $f$ on $X$ with $\|f\|_{\mathcal{L}^{p, \omega, \theta}(X)}<\infty$ is denoted by $\mathcal{L}^{p, \omega, \theta}(X)$. The space $\mathcal{L}^{p, \omega, \theta}(X)$ is called a Morrey space of an integral form. Here note that $2 d_{X}$ can be replaced by $\kappa d_{X}$ with $\kappa>1$.

Our aim in this paper is to give a general version of Sobolev-type inequality for Riesz potentials $I_{\alpha, \tau} f$ of functions in Morrey spaces $\mathcal{L}^{p, \omega, \theta}(X)$ of an integral form over nondoubling metric measure spaces $X$ (see Theorem 3.3), as an extension of [15, Theorem 5.4] in the Euclidean case. Our results are new even for the doubling metric measure setting. To this end, we apply Hedberg's trick [8] by the use of the Hardy-Littlewood maximal operator $M_{\lambda}$ adapted to our setting (see Theorem 2.4). See Section 2 for the definition of $M_{\lambda}$ and Remarks 2.1 and 2.2 on the number $\lambda$.

\section{Boundedness of the maximal operator}

Throughout the paper, we let $C$ denote various constants independent of the variables in question and $C(a, b, \ldots)$ be a constant that depends on $a, b, \ldots$ only.

For a locally integrable function $f$ on $X$ and $\lambda \geq 1$, the Hardy-Littlewood maximal function $M_{\lambda} f$ is defined by

$$
M_{\lambda} f(x)=\sup _{r>0} \frac{1}{\mu(B(x, \lambda r))} \int_{B(x, r)}|f(y)| d \mu(y) .
$$

For $\lambda \geq 1$, we say that $X$ satisfies $(M \lambda)$ if there exists a constant $C>0$ such that

$$
\mu\left(\left\{x \in X: M_{\lambda} f(x)>k\right\}\right) \leq \frac{C}{k} \int_{X}|f(y)| d \mu(y)
$$

for all measurable functions $f \in L^{1}(X)$ and $k>0$.

Remark 2.1. In (2.1), we can not reduce the number $\lambda$ any more (Stempak 27]).

As for the precise value of $\lambda$, we know the following.

Remark 2.2. By a covering argument, Nazarov, Treil and Volberg 19,20 proved that $X$ satisfies (M3) if $X$ is a separable metric space. Meanwhile $X$ satisfies $(M \lambda)$ for any $\lambda>0$ if $\mu$ satisfies the doubling condition (see 9]). Terasawa 29 showed that $X$ satisfies $(M \lambda)$ 
for $\lambda \geq 2$ if $\mu(B(x, r))$ is continuous in the variable $r>0$ when $x \in X$ is fixed. In [24], Sawano showed that $X$ satisfies $(M \lambda)$ for $\lambda \geq 2$ if $X$ is a separable metric space. Another remarkable example of the Poincaré disc can be found in [28] where 2.1] with $\lambda=1$ is established despite the fact that the corresponding Riemannian volume $\mu$ is non-doubling. As in [7, Appendix], the Euclidean space $\mathbf{R}^{N}$, endowed with $\mu \equiv e^{|x|^{2}} d x$, fails to satisfy $(M 1)$.

We know the following result.

Lemma 2.3. Let $1<p_{0}<\infty$ and let $\lambda \geq 1$. Suppose $X$ satisfies $(M \lambda)$. Then there exists a constant $C>0$ such that

$$
\int_{X}\left\{M_{\lambda} f(x)\right\}^{p_{0}} d \mu(x) \leq C
$$

for all measurable functions $f$ on $X$ with $\|f\|_{L^{p_{0}(X)}} \leq 1$.

Now we are ready to show the boundedness of the maximal operator $M_{\lambda}$.

Theorem 2.4. Let $1 \leq \theta_{1}<\theta_{2}$ and $\lambda>\theta_{1}\left(\theta_{2}+1\right) /\left(\theta_{2}-\theta_{1}\right)$. Assume that $X$ satisfies $(M \lambda)$. Further suppose

$\left(\omega 1^{\prime}\right) r \mapsto r^{-\varepsilon_{1}} \omega(r)$ is almost increasing in $\left(0, d_{X}\right]$ for some $\varepsilon_{1}>0$.

If $p>1$, then there is a constant $C>0$ such that

$$
\left\|M_{\lambda} f\right\|_{\mathcal{L}^{p, \omega, \theta_{2}(X)}} \leq C\|f\|_{\mathcal{L}^{p, \omega, \theta_{1}(X)}}
$$

for all $f \in \mathcal{L}^{p, \omega, \theta_{1}}(X)$.

Proof. Let $f$ be a nonnegative measurable function on $X$ with $\|f\|_{\mathcal{L}^{p, \omega, \theta_{1}(X)}} \leq 1$. Let $z \in X$ and $0<r \leq 2 d_{X}$. For $\kappa_{1}=\theta_{2} / \theta_{1}>1$, write

$$
f(y)=f(y) \chi_{B\left(z, \kappa_{1} r\right)}(y)+f(y) \chi_{X \backslash B\left(z, \kappa_{1} r\right)}(y):=f_{1}(y)+f_{2}(y)
$$

where $\chi_{E}$ is the characteristic function of $E$.

By Lemma 2.3 and $(\omega 2)$, we have

$$
\begin{aligned}
& \int_{0}^{2 d_{X}} \frac{\omega(r)}{\mu\left(B\left(z, \theta_{2} r\right)\right)}\left(\int_{B(z, r)}\left\{M_{\lambda} f_{1}(x)\right\}^{p} d \mu(y)\right) \frac{d r}{r} \\
\leq & \int_{0}^{2 d_{X}} \frac{\omega(r)}{\mu\left(B\left(z, \theta_{2} r\right)\right)}\left(\int_{X}\left\{M_{\lambda} f_{1}(x)\right\}^{p} d \mu(y)\right) \frac{d r}{r} \\
\leq & C \int_{0}^{2 d_{X}} \frac{\omega(r)}{\mu\left(B\left(z, \theta_{2} r\right)\right)}\left(\int_{X} f_{1}(y)^{p} d \mu(y)\right) \frac{d r}{r}
\end{aligned}
$$




$$
\begin{aligned}
& \leq C \int_{0}^{2 d_{X}} \frac{\omega(r)}{\mu\left(B\left(z, \theta_{2} r\right)\right)}\left(\int_{B\left(z, \kappa_{1} r\right)} f(y)^{p} d \mu(y)\right) \frac{d r}{r} \\
& \leq C \int_{0}^{2 \kappa_{1} d_{X}} \frac{\omega\left(\kappa_{1}^{-1} r\right)}{\mu\left(B\left(z, \theta_{2} \kappa_{1}^{-1} r\right)\right)}\left(\int_{B(z, r)} f(y)^{p} d \mu(y)\right) \frac{d r}{r} \\
& \leq C \int_{0}^{2 d_{X}} \frac{\omega(r)}{\mu\left(B\left(z, \theta_{1} r\right)\right)}\left(\int_{B(z, r)} f(y)^{p} d \mu(y)\right) \frac{d r}{r} \\
& \leq C .
\end{aligned}
$$

Next we treat $f_{2}$. Let

$$
\kappa_{2}=\lambda\left(1-\frac{1}{\kappa_{1}}\right)-\frac{1}{\kappa_{1}}=\lambda\left(1-\frac{\theta_{1}}{\theta_{2}}\right)-\frac{\theta_{1}}{\theta_{2}} .
$$

Then note that $\kappa_{2}>\theta_{1}$ and

$$
B\left(z, \kappa_{2} d(z, y)\right) \subset B(x, \lambda d(x, y))
$$

for $x \in B(z, r)$ and $y \in X \backslash B\left(z, \kappa_{1} r\right)$. Indeed, when $w \in B\left(z, \kappa_{2} d(z, y)\right)$, we have

$$
\begin{aligned}
d(w, x) & \leq d(w, z)+d(z, x)<\kappa_{2} d(z, y)+\frac{1}{\kappa_{1}} d(z, y)=\lambda\left(1-\frac{1}{\kappa_{1}}\right) d(z, y) \\
& \leq \lambda\left(1-\frac{1}{\kappa_{1}}\right)\left(1-\frac{1}{\kappa_{1}}\right)^{-1} d(x, y)=\lambda d(x, y)
\end{aligned}
$$

For $\gamma=\kappa_{2} \theta_{1}^{-1}>1$, let $j_{0}$ be the smallest integer $\kappa_{1} \gamma^{j_{0} / 2} r \geq d_{X}$. For $x \in B(z, r)$ and $0<\varepsilon<\varepsilon_{1}$, we see from Hölder's inequality and 2.3 that

$$
\begin{aligned}
M_{\lambda} f_{2}(x) & =\sup _{\rho>0} \frac{1}{\mu(B(x, \lambda \rho))} \int_{B(x, \rho)} f_{2}(y) d \mu(y) \\
& \leq \sup _{\rho>0}\left(\frac{1}{\mu(B(x, \lambda \rho))} \int_{B(x, \rho)} f_{2}(y)^{p} d \mu(y)\right)^{1 / p} \\
& \leq C\left(\int_{X \backslash B\left(z, \kappa_{1} r\right)} \frac{1}{\mu(B(x, \lambda d(x, y)))} f(y)^{p} d \mu(y)\right)^{1 / p} \\
& \leq C\left(\int_{X \backslash B\left(z, \kappa_{1} r\right)} \frac{1}{\mu\left(B\left(z, \kappa_{2} d(z, y)\right)\right)} f(y)^{p} d \mu(y)\right)^{1 / p} .
\end{aligned}
$$

We decompose

$$
M_{\lambda} f_{2}(x) \leq C\left(\sum_{j=1}^{j_{0}} \int_{B\left(z, \kappa_{1} \gamma^{j / 2} r\right) \backslash B\left(z, \kappa_{1} \gamma^{(j-1) / 2} r\right)} \frac{1}{\mu\left(B\left(z, \kappa_{2} d(z, y)\right)\right)} f(y)^{p} d \mu(y)\right)^{1 / p}
$$




$$
\begin{aligned}
& \leq C \sum_{j=1}^{j_{0}}\left(\int_{B\left(z, \kappa_{1} \gamma^{j / 2} r\right) \backslash B\left(z, \kappa_{1} \gamma^{(j-1) / 2} r\right)} \frac{1}{\mu\left(B\left(z, \kappa_{2} d(z, y)\right)\right)} f(y)^{p} d \mu(y)\right)^{1 / p} \\
& \leq C \sum_{j=1}^{j_{0}}\left(\frac{1}{\mu\left(B\left(z, \kappa_{1} \kappa_{2} \gamma^{(j-1) / 2} r\right)\right)} \int_{B\left(z, \kappa_{1} \gamma^{j / 2} r\right)} f(y)^{p} d \mu(y)\right)^{1 / p} \\
& =C \sum_{j=1}^{j_{0}}\left(\frac{1}{\mu\left(B\left(z, \kappa_{1} \gamma^{(j+1) / 2} \theta_{1} r\right)\right)} \int_{B\left(z, \kappa_{1} \gamma^{j / 2} r\right)} f(y)^{p} d \mu(y)\right)^{1 / p} \\
& \leq C\left(\sum_{j=1}^{j_{0}}\left(\left(\kappa_{1} \gamma^{j / 2} r\right)^{\varepsilon / p} \omega\left(\kappa_{1} \gamma^{j / 2} r\right)^{-1 / p}\right)^{p^{\prime}}\right)^{1 / p^{\prime}} \\
& \quad \times\left(\sum_{j=1}^{j_{0}} \frac{\left(\kappa_{1} \gamma^{j / 2} r\right)^{-\varepsilon} \omega\left(\kappa_{1} \gamma^{j / 2} r\right)}{\mu\left(B\left(z, \kappa_{1} \gamma^{(j+1) / 2} \theta_{1} r\right)\right)} \int_{B\left(z, \kappa_{1} \gamma^{j / 2} r\right)} f(y)^{p} d \mu(y)\right)^{1 / p}
\end{aligned}
$$

by Hölder's inequality, where $1 / p+1 / p^{\prime}=1$. Here note from $\left(\omega 1^{\prime}\right)$ that

$$
\begin{aligned}
& \left(\sum_{j=1}^{j_{0}}\left(\left(\kappa_{1} \gamma^{j / 2} r\right)^{\varepsilon / p} \omega\left(\kappa_{1} \gamma^{j / 2} r\right)^{-1 / p}\right)^{p^{\prime}}\right)^{1 / p^{\prime}} \\
\leq & C r^{\varepsilon_{1} / p} \omega(r)^{-1 / p}\left(\sum_{j=1}^{j_{0}}\left(\kappa_{1} \gamma^{j / 2} r\right)^{\left(\varepsilon / p-\varepsilon_{1} / p\right) p^{\prime}}\right)^{1 / p^{\prime}} \leq C r^{\varepsilon / p} \omega(r)^{-1 / p}
\end{aligned}
$$

for $0<\varepsilon<\varepsilon_{1}$. Further note from $(\omega 2)$ that

$$
\begin{aligned}
& \sum_{j=1}^{j_{0}} \frac{\left(\kappa_{1} \gamma^{j / 2} r\right)^{-\varepsilon} \omega\left(\kappa_{1} \gamma^{j / 2} r\right)}{\mu\left(B\left(z, \kappa_{1} \gamma^{(j+1) / 2} \theta_{1} r\right)\right)} \int_{B\left(z, \kappa_{1} \gamma^{j / 2} r\right)} f(y)^{p} d \mu(y) \\
\leq & C \sum_{j=1}^{j_{0}} \int_{\kappa_{1} \gamma^{j / 2} r}^{\kappa_{1} \gamma^{(j+1) / 2} r} \frac{t^{-\varepsilon} \omega(t)}{\mu\left(B\left(z, \theta_{1} t\right)\right)}\left(\int_{B(z, t)} f(y)^{p} d \mu(y)\right) \frac{d t}{t} \\
\leq & C \int_{\kappa_{1} \gamma^{1 / 2} r}^{\gamma d_{X}} \frac{t^{-\varepsilon} \omega(t)}{\mu\left(B\left(z, \theta_{1} t\right)\right)}\left(\int_{B(z, t)} f(y)^{p} d \mu(y)\right) \frac{d t}{t} \\
\leq & C \int_{r}^{2 d_{X}} \frac{t^{-\varepsilon} \omega(t)}{\mu\left(B\left(z, \theta_{1} t\right)\right)}\left(\int_{B(z, t)} f(y)^{p} d \mu(y)\right) \frac{d t}{t} .
\end{aligned}
$$

By (2.4), (2.5) and (2.6), we have

$$
M_{\lambda} f_{2}(x) \leq C r^{\varepsilon / p} \omega(r)^{-1 / p}\left(\int_{r}^{2 d_{X}} \frac{t^{-\varepsilon} \omega(t)}{\mu\left(B\left(z, \theta_{1} t\right)\right)}\left(\int_{B(z, t)} f(y)^{p} d \mu(y)\right) \frac{d t}{t}\right)^{1 / p}
$$


Hence, we obtain

$$
\begin{aligned}
& \int_{0}^{2 d_{X}} \frac{\omega(r)}{\mu\left(B\left(z, \theta_{2} r\right)\right)}\left(\int_{B(z, r)}\left\{M_{\lambda} f_{2}(x)\right\}^{p} d \mu(y)\right) \frac{d r}{r} \\
\leq & C \int_{0}^{2 d_{X}} r^{\varepsilon}\left(\int_{r}^{2 d_{X}} \frac{t^{-\varepsilon} \omega(t)}{\mu\left(B\left(z, \theta_{1} t\right)\right)}\left(\int_{B(z, t)} f(y)^{p} d \mu(y)\right) \frac{d t}{t}\right) \frac{d r}{r} \\
\leq & C \int_{0}^{2 d_{X}} \frac{t^{-\varepsilon} \omega(t)}{\mu\left(B\left(z, \theta_{1} t\right)\right)}\left(\int_{B(z, t)} f(y)^{p} d \mu(y)\right)\left(\int_{0}^{t} r^{\varepsilon} \frac{d r}{r}\right) \frac{d t}{t} \\
\leq & C \int_{0}^{2 d_{X}} \frac{\omega(t)}{\mu\left(B\left(z, \theta_{1} t\right)\right)}\left(\int_{B(z, t)} f(y)^{p} d \mu(y)\right) \frac{d t}{t} \\
\leq & C .
\end{aligned}
$$

Thus, in view of $(2.2)$ and $(2.7)$, we complete the proof.

Remark 2.5. Note that $\left(\omega 1^{\prime}\right)$ implies $(\omega 1)$. Let $\omega(r)=r^{\sigma}(\log (e+1 / r))^{\beta}$ be as in Example 1.1. Then note that $\left(\omega 1^{\prime}\right)$ holds for $0<\varepsilon_{1}<\sigma$.

\section{Sobolev-type inequality}

We recall the following lemma.

Lemma 3.1. 11, Lemma 5.1(3)] Set

$$
\omega^{-1}(r)=\sup \{s>0 \mid \omega(s)<r\}
$$

for $r>0$. Then

$$
\omega\left(\omega^{-1}(r)\right)=r
$$

for all $r>0$ with $\omega^{-1}(r)<\infty$.

We consider the following condition:

$(\omega \alpha)$ for $\alpha>0$, there exist constants $\varepsilon_{2}>0$ and $A_{1} \geq 1$ such that

$$
r_{2}^{\varepsilon_{2}+\alpha} \omega\left(r_{2}\right)^{-1 / p} \leq A_{1} r_{1}^{\varepsilon_{2}+\alpha} \omega\left(r_{1}\right)^{-1 / p}
$$

whenever $0<r_{1}<r_{2}<d_{X}$.

Lemma 3.2. Let $1 \leq \theta<\tau$. Assume that $(\omega \alpha)$ holds. Then there exists a constant $C>0$ such that

$$
\int_{X \backslash B(x, \delta)} \frac{d(x, y)^{\alpha} f(y)}{\mu(B(x, \tau d(x, y)))} d \mu(y) \leq C \delta^{\alpha} \omega(\delta)^{-1 / p}
$$

for all $x \in X, 0<\delta<d_{X} / 2$ and nonnegative $f \in \mathcal{L}^{p, \omega, \theta}(X)$ with $\|f\|_{\mathcal{L}^{p, \omega, \theta}(X)} \leq 1$. 
Proof. Let $f$ be a nonnegative measurable function with $\|f\|_{\mathcal{L}^{p, \omega, \theta}(X)} \leq 1$. Let $x \in X$ and $0<\delta<d_{X} / 2$. We find by $(\omega \alpha)$

$$
\begin{aligned}
& \int_{X \backslash B(x, \delta)} \frac{d(x, y)^{\alpha} f(y)}{\mu(B(x, \tau d(x, y)))} d \mu(y) \\
\leq & \int_{X \backslash B(x, \delta)} \frac{d(x, y)^{\alpha}}{\mu(B(x, \tau d(x, y)))} \omega(d(x, y))^{-1 / p} d \mu(y) \\
& +\int_{X \backslash B(x, \delta)} \frac{d(x, y)^{\alpha} f(y)}{\mu(B(x, \tau d(x, y)))} \cdot \frac{f(y)^{p-1}}{\omega(d(x, y))^{-(p-1) / p}} d \mu(y) \\
\leq & \int_{X \backslash B(x, \delta)} \frac{d(x, y)^{\alpha}}{\mu(B(x, \tau d(x, y)))} \omega(d(x, y))^{-1 / p} d \mu(y) \\
& +C \delta^{\alpha} \omega(\delta)^{-1 / p} \int_{X \backslash B(x, \delta)} \frac{\omega(d(x, y))}{\mu(B(x, \tau d(x, y)))} f(y)^{p} d \mu(y) \\
= & I_{1}+C I_{2} .
\end{aligned}
$$

Let $j_{0}$ be the smallest integer such that $\tau^{j_{0}} \delta \geq d_{X}$. By $(\omega 1),(\omega 2)$ and $(\omega \alpha)$, we have

$$
\begin{aligned}
I_{1} & =\sum_{j=1}^{j_{0}} \int_{B\left(x, \tau^{j} \delta\right) \backslash B\left(x, \tau^{j-1} \delta\right)} \frac{d(x, y)^{\alpha}}{\mu(B(x, \tau d(x, y)))} \omega(d(x, y))^{-1 / p} d \mu(y) \\
& \leq C \sum_{j=1}^{j_{0}}\left(\tau^{j} \delta\right)^{\alpha} \omega\left(\tau^{j} \delta\right)^{-1 / p} \leq C \int_{\delta}^{\tau d_{X}} \rho^{\alpha} \omega(\rho)^{-1 / p} \frac{d \rho}{\rho} \\
& \leq C \int_{\delta}^{d_{X}} \rho^{\alpha} \omega(\rho)^{-1 / p} \frac{d \rho}{\rho} \leq C \delta^{\alpha} \omega(\delta)^{-1 / p} .
\end{aligned}
$$

Next, for $\gamma=\tau \theta^{-1}>1$, let $j_{1}$ be the smallest positive integer such that $\gamma^{j_{1} / 2} \delta \geq d_{X}$. Then we have by $(\omega 1)$ and $(\omega 2)$

$$
\begin{aligned}
I_{2} & =C \delta^{\alpha} \omega(\delta)^{-1 / p} \sum_{j=1}^{j_{1}} \int_{B\left(x, \gamma^{j / 2} \delta\right) \backslash B\left(x, \gamma^{(j-1) / 2} \delta\right)} \frac{\omega(d(x, y))}{\mu(B(x, \tau d(x, y)))} f(y)^{p} d \mu(y) \\
& \leq C \delta^{\alpha} \omega(\delta)^{-1 / p} \sum_{j=1}^{j_{1}} \frac{\omega\left(\gamma^{j / 2} \delta\right)}{\mu\left(B\left(x, \gamma^{(j+1) / 2} \theta \delta\right)\right)} \int_{B\left(x, \gamma^{j / 2} \delta\right)} f(y)^{p} d \mu(y) \\
& \leq C \delta^{\alpha} \omega(\delta)^{-1 / p} \sum_{j=1}^{j_{1}} \int_{\gamma^{j / 2} \delta}^{\gamma^{(j+1) / 2} \delta} \frac{\omega(t)}{\mu(B(x, \theta t))}\left(\int_{B(x, t)} f(y)^{p} d \mu(y)\right) \frac{d t}{t}
\end{aligned}
$$

Hence

$$
\begin{aligned}
I_{2} & \leq C \delta^{\alpha} \omega(\delta)^{-1 / p} \int_{\gamma^{1 / 2} \delta}^{\gamma d_{X}} \frac{\omega(t)}{\mu(B(x, \theta t))}\left(\int_{B(x, t)} f(y)^{p} d \mu(y)\right) \frac{d t}{t} \\
& \leq C \delta^{\alpha} \omega(\delta)^{-1 / p} \int_{0}^{2 d_{X}} \frac{\omega(t)}{\mu(B(x, \theta t))}\left(\int_{B(x, t)} f(y)^{p} d \mu(y)\right) \frac{d t}{t} \\
& \leq C \delta^{\alpha} \omega(\delta)^{-1 / p}
\end{aligned}
$$


since we have by $(\omega 1)$ and $(\omega 2)$

$$
\begin{aligned}
& \int_{2 d_{X}}^{\gamma d_{X}} \frac{\omega(t)}{\mu(B(x, \theta t))}\left(\int_{B(x, t)} f(y)^{p} d \mu(y)\right) \frac{d t}{t} \\
\leq & C \frac{\omega\left(d_{X}\right)}{\mu(X)} \int_{X} f(y)^{p} d \mu(y) \\
\leq & C \int_{d_{X}}^{2 d_{X}} \frac{\omega(t)}{\mu(B(x, \theta t))}\left(\int_{B(x, t)} f(y)^{p} d \mu(y)\right) \frac{d t}{t} \\
\leq & C \int_{0}^{2 d_{X}} \frac{\omega(t)}{\mu(B(x, \theta t))}\left(\int_{B(x, t)} f(y)^{p} d \mu(y)\right) \frac{d t}{t}
\end{aligned}
$$

when $\gamma>2$. Thus we obtain the required result.

Before stating the main theorem we give the assumptions for the function in Sobolevtype inequalities. We consider a function

$$
\Psi(t):[0, \infty) \rightarrow[0, \infty)
$$

satisfies

$(\Psi 1) \Psi(\cdot)$ is continuous on $[0, \infty)$;

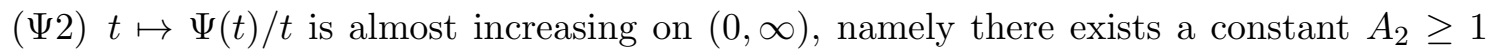
such that

$$
\Psi\left(t_{1}\right) / t_{1} \leq A_{2} \Psi\left(t_{2}\right) / t_{2} \quad \text { whenever } 0<t_{1}<t_{2}
$$

( $\Psi 3$ ) there exists a constant $A_{3} \geq 1$ such that

$$
\Psi\left(t \omega^{-1}\left(t^{-p}\right)^{\alpha}\right) \leq A_{3} t^{p} \quad \text { for all } t \geq 1 .
$$

We write $\bar{\psi}(t)=\sup _{0<s \leq t}(\Psi(s) / s)$ and

$$
\bar{\Psi}(t)=\int_{0}^{t} \bar{\psi}(r) d r
$$

for $t \geq 0$. Then $\bar{\Psi}(\cdot)$ is convex and

$$
\Psi(t / 2) \leq \bar{\Psi}(t) \leq A_{2} \Psi(t)
$$

for all $t \geq 0$.

Let $\theta \geq 1$. Given $\Psi(t)$ and $\omega(r)$ as above, we define the $\mathcal{L}^{\Psi, \omega, \theta}$ norm by

$$
\begin{aligned}
&\|f\|_{\mathcal{L}^{\Psi, \omega, \theta}(X)}=\inf \{\lambda>0 ; \\
&\left.\sup _{x \in X}\left(\int_{0}^{2 d_{X}} \frac{\omega(r)}{\mu(B(x, \theta r))}\left(\int_{B(x, r)} \bar{\Psi}(|f(y)| / \lambda) d \mu(y)\right) \frac{d r}{r}\right) \leq 1\right\} .
\end{aligned}
$$


The space of all measurable functions $f$ on $X$ with $\|f\|_{\mathcal{L}^{\Psi, \omega, \theta}(X)}<\infty$ is denoted by $\mathcal{L}^{\Psi, \omega, \theta}(X)$.

As an application of $M_{\lambda}$, we establish a Sobolev-type inequality for $I_{\alpha, \tau} f$ of functions in $\mathcal{L}^{p, \omega, \theta_{1}}(X)$ in the non-doubling setting.

Theorem 3.3. Let $X$ be a non-doubling metric measure space. Let $1 \leq \theta_{1}<\theta_{2}$ and $\theta_{1}\left(\theta_{2}+1\right) /\left(\theta_{2}-\theta_{1}\right)<\lambda<\tau$. Assume that $X$ satisfies $(M \lambda)$ and $\left(\omega 1^{\prime}\right)$ and $(\omega \alpha)$ hold. If $p>1$, then there exists a constant $C>0$ such that

$$
\left\|I_{\alpha, \tau} f\right\|_{\mathcal{L}^{\Psi, \omega, \theta_{2}(X)}} \leq C\|f\|_{\mathcal{L}^{p, \omega, \theta_{1}}(X)}
$$

for all $f \in \mathcal{L}^{p, \omega, \theta_{1}}(X)$.

Proof. Let $f$ be a nonnegative measurable function on $X$ such that $\|f\|_{\mathcal{L}^{p, \omega, \theta_{1}(X)}} \leq 1$. Let $x \in X$ and $0<\delta<d_{X} / 2$. If $M_{\lambda} f(x)<1$, then

$$
I_{\alpha, \tau} f(x)=\int_{B\left(x, d_{X}\right)} \frac{d(x, y)^{\alpha} f(y)}{\mu(B(x, \tau d(x, y)))} d \mu(y) \leq C d_{X}^{\alpha} M_{\lambda} f(x) \leq C
$$

by $\lambda<\tau$ (see 21, p. 134]). If $\omega^{-1}\left(\left\{M_{\lambda} f(x)\right\}^{-p}\right) \geq d_{X} / 2$ and $M_{\lambda} f(x) \geq 1$, then

$$
\begin{aligned}
I_{\alpha, \tau} f(x) & =\int_{B\left(x, d_{X}\right)} \frac{d(x, y)^{\alpha} f(y)}{\mu(B(x, \tau d(x, y)))} d \mu(y) \chi_{\left\{y \in X: M_{\lambda} f(y) \geq 1\right\}}(x) \\
& \leq C d_{X}^{\alpha} M_{\lambda} f(x) \chi_{\left\{y \in X: M_{\lambda} f(y) \geq 1\right\}}(x) \\
& \leq C M_{\lambda} f(x) \omega^{-1}\left(\left\{M_{\lambda} f(x)\right\}^{-p}\right)^{\alpha} \chi_{\left\{y \in X: M_{\lambda} f(y) \geq 1\right\}}(x) .
\end{aligned}
$$

By Lemma 3.2, we find

$$
\begin{aligned}
I_{\alpha, \tau} f(x) & =\int_{B(x, \delta)} \frac{d(x, y)^{\alpha} f(y)}{\mu(B(x, \tau d(x, y)))} d \mu(y)+\int_{X \backslash B(x, \delta)} \frac{d(x, y)^{\alpha} f(y)}{\mu(B(x, \tau d(x, y)))} d \mu(y) \\
& \leq C\left\{\delta^{\alpha} M_{\lambda} f(x)+\delta^{\alpha} \omega(\delta)^{-1 / p}\right\} .
\end{aligned}
$$

If $\omega^{-1}\left(\left\{M_{\lambda} f(x)\right\}^{-p}\right)<d_{X} / 2$ and $M_{\lambda} f(x) \geq 1$, then take $\delta=\omega^{-1}\left(\left\{M_{\lambda} f(x)\right\}^{-p}\right)$. Then we have

$$
I_{\alpha, \tau} f(x) \leq C M_{\lambda} f(x) \omega^{-1}\left(\left\{M_{\lambda} f(x)\right\}^{-p}\right)^{\alpha} \chi_{\left\{y \in X: M_{\lambda} f(y) \geq 1\right\}}(x)
$$

by Lemma 3.1. Therefore we obtain

$$
I_{\alpha, \tau} f(x) \leq C_{1}^{\prime} \max \left\{M_{\lambda} f(x) \omega^{-1}\left(\left\{M_{\lambda} f(x)\right\}^{-p}\right)^{\alpha} \chi_{\left\{y \in X: M_{\lambda} f(y) \geq 1\right\}}(x), 1\right\},
$$

so that by $(\Psi 2)$ and $(\Psi 3)$, we have

$$
\begin{aligned}
\Psi\left(I_{\alpha, \tau} f(x) / C_{1}^{\prime}\right) & \leq C\left\{\Psi\left(M_{\lambda} f(x) \omega^{-1}\left(\left\{M_{\lambda} f(x)\right\}^{-p}\right)^{\alpha}\right) \chi_{\left\{y \in X: M_{\lambda} f(y) \geq 1\right\}}(x)+1\right\} \\
& \leq C\left[\left\{M_{\lambda} f(x)\right\}^{p}+1\right] .
\end{aligned}
$$


Therefore we obtain by Theorem 2.4 .

$$
\begin{aligned}
& \int_{0}^{2 d_{X}} \frac{\omega(r)}{\mu\left(B\left(z, \theta_{2} r\right)\right)}\left(\int_{B(z, r)} \Psi\left(I_{\alpha, \tau} f(x) / C_{1}^{\prime}\right) d \mu(x)\right) \frac{d r}{r} \\
\leq & C\left\{\int_{0}^{2 d_{X}} \frac{\omega(r)}{\mu\left(B\left(z, \theta_{2} r\right)\right)}\left(\int_{B(z, r)}\left\{M_{\lambda} f(x)\right\}^{p} d \mu(x)\right) \frac{d r}{r}+\int_{0}^{2 d_{X}} \omega(r) \frac{d r}{r}\right\} \\
\leq & C
\end{aligned}
$$

for all $z \in X$ since

$$
\int_{0}^{2 d_{X}} \omega(r) \frac{d r}{r}=\int_{0}^{2 d_{X}} r^{-\varepsilon_{1}} \omega(r) \cdot r^{\varepsilon_{1}} \frac{d r}{r} \leq C \int_{0}^{2 d_{X}} r^{\varepsilon_{1}} \frac{d r}{r} \leq C
$$

by $\left(\omega 1^{\prime}\right)$ and $(\omega 3)$. This completes the proof of the theorem.

As in the proof of Theorem 3.3, we can prove the following theorem for the doubling metric measure case.

Theorem 3.4. Let $X$ be a doubling metric measure space. Assume that $\left(\omega 1^{\prime}\right)$ and $(\omega \alpha)$ hold. If $p>1$, then there exists a constant $C>0$ such that

$$
\left\|I_{\alpha, 1} f\right\|_{\mathcal{L}^{\Psi, \omega, 1}(X)} \leq C\|f\|_{\mathcal{L}^{p, \omega, 1}(X)}
$$

for all $f \in \mathcal{L}^{p, \omega, 1}(X)$.

\section{Corollaries}

In this section, we give consequences of Theorems 3.3 and 3.4 .

Let

$$
\omega(r)=r^{\sigma}(\log (e+1 / r))^{\beta}
$$

be as in Example 1.1 and set

$$
\Psi(t)=\left\{t(\log (e+t))^{\alpha \beta / \sigma}\right\}^{p^{*}}
$$

where $1 / p^{*}=1 / p-\alpha / \sigma$. If $1<p<\sigma / \alpha$, then $\omega(r)$ satisfies condition $(\omega \alpha)$ and $\Psi(t)$ satisfies condition $(\Psi 3)$.

Example 4.1. Let $X_{1}=\left\{(x, 0) \in \mathbf{R}^{2}: 0 \leq x<1\right\}$ and $X_{2}=\left\{(x, y) \in \mathbf{R}^{2}:|x|<1, x_{1}<\right.$ $0\}$ and define $(X, d, \mu)=\left(X_{1}, d_{2}, m_{1}\right) \cup\left(X_{2}, d_{2}, m_{2}\right)$, where $d_{2}$ denotes the 2-dimensional Euclidean distance and $m_{i}$ denotes the $i$-dimensional Lebesgue measure. It is easy to show that $\mu$ is non-doubling. Since $X$ is a separable metric space, $X$ satisfies $(M \lambda)$ for $\lambda \geq 2$ (see Remark 2.2). 
Let $\theta \geq 1$ and $p \geq 1$. Consider the function

$$
f(y)=d_{2}(0, y)^{-a} \chi_{X_{2}}(y)
$$

for $a<\min \{2 / p, \sigma / p\}$. Then note that

$$
\begin{aligned}
& \int_{0}^{4} \frac{\omega(r)}{\mu(B(x, \theta r))}\left(\int_{B(x, r)}|f(y)|^{p} d \mu(y)\right) \frac{d r}{r} \\
\leq & C \int_{0}^{4} \frac{\omega(r)}{\mu(B(x, \theta r))}\left(\int_{B(0, r) \cap X_{2}}|f(y)|^{p} d \mu(y)\right) \frac{d r}{r} \\
\leq & C \int_{0}^{4} \frac{\omega(r)}{\mu(B(x, \theta r))} r^{2-a p} \frac{d r}{r} \\
\leq & C \int_{0}^{4} r^{\sigma-a p}(\log (e+1 / r))^{\beta} \frac{d r}{r}<\infty
\end{aligned}
$$

for all $x \in X$ since $\mu(B(x, \theta r)) \geq C r^{2}$ for all $x \in X$ and $0<r<4$. Therefore $f \in$ $\mathcal{L}^{p, \omega, \theta}(X)$, so that $\mathcal{L}^{p, \omega, \theta}(X) \neq\{0\}$.

Corollary 4.2. Let $X$ be a non-doubling metric measure space. Let $1 \leq \theta_{1}<\theta_{2}$ and $\theta_{1}\left(\theta_{2}+1\right) /\left(\theta_{2}-\theta_{1}\right)<\lambda<\tau$. Assume that $X$ satisfies $(M \lambda)$. If $1<p<\sigma / \alpha$, then there exists a constant $C>0$ such that

$$
\left\|I_{\alpha, \tau} f\right\|_{\mathcal{L}^{\Psi, \omega, \theta_{2}(X)}} \leq C\|f\|_{\mathcal{L}^{p, \omega, \theta_{1}}(X)}
$$

for all $f \in \mathcal{L}^{p, \omega, \theta_{1}}(X)$.

Corollary 4.3. Let $X$ be a doubling metric measure space. If $1<p<\sigma / \alpha$, then there exists a constant $C>0$ such that

$$
\left\|I_{\alpha, 1} f\right\|_{\mathcal{L}^{\Psi, \omega, 1}(X)} \leq C\|f\|_{\mathcal{L}^{p, \omega, 1}(X)}
$$

for all $f \in \mathcal{L}^{p, \omega, 1}(X)$.

\section{References}

[1] D. R. Adams, A note on Riesz potentials, Duke Math. J. 42 (1975), no. 4, 765-778.

[2] D. R. Adams and L. I. Hedberg, Function Spaces and Potential Theory, Grundlehren der mathematischen Wissenschaften 314, Springer-Verlag, Berlin, 1996.

[3] A. Björn and J. Björn, Nonlinear Potential Theory on Metric Spaces, EMS Tracts in Mathematics 17, European Mathematical Society (EMS), Zürich, 2011. 
[4] F. Chiarenza and M. Frasca, Morrey spaces and Hardy-Littlewood maximal function, Rend. Mat. Appl. (7) 7 (1987), no. 3-4, 273-279.

[5] D. Cruz-Uribe and P. Shukla, The boundedness of fractional maximal operators on variable Lebesgue spaces over spaces of homogeneous type, Studia Math. 242 (2018), no. 2, 109-139.

[6] P. Hajłasz and P. Koskela, Sobolev met Poincaré, Mem. Amer. Math. Soc. 145 (2000), no. 688,101 pp.

[7] D. Hashimoto, Y. Sawano and T. Shimomura, Gagliardo-Nirenberg inequality for generalized Riesz potentials of functions in Musielak-Orlicz spaces over quasi-metric measure spaces, Colloq. Math. 161 (2020), no. 1, 51-66.

[8] L. I. Hedberg, On certain convolution inequalities, Proc. Amer. Math. Soc. 36 (1972), 505-510.

[9] J. Heinonen, Lectures on Analysis on Metric Spaces, Universitext, Springer-Verlag, New York, 2001.

[10] A. Kairema, Two-weight norm inequalities for potential type and maximal operators in a metric space, Publ. Mat. 57 (2013), no. 1, 3-56.

[11] F.-Y. Maeda, Y. Mizuta, T. Ohno and T. Shimomura, Boundedness of maximal operators and Sobolev's inequality on Musielak-Orlicz-Morrey spaces, Bull. Sci. Math. 137 (2013), no. 1, 76-96.

[12] Y. Mizuta, E. Nakai, T. Ohno and T. Shimomura, An elementary proof of Sobolev embeddings for Riesz potentials of functions in Morrey spaces $L^{1, \nu, \beta}(G)$, Hiroshima Math. J. 38 (2008), no. 3, 425-436.

[13] _ Maximal functions, Riesz potentials and Sobolev embeddings on MusielakOrlicz-Morrey spaces of variable exponent in $\mathbf{R}^{n}$, Rev. Mat. Complut. 25 (2012), no. 2, 413-434.

[14] Y. Mizuta and T. Shimomura, Sobolev embeddings for Riesz potentials of functions in Morrey spaces of variable exponent, J. Math. Soc. Japan 60 (2008), no. 2, 583-602.

[15] _ Sobolev's inequality for Riesz potentials of functions in Morrey spaces of integral form, Math. Nachr. 283 (2010), no. 9, 1336-1352.

[16] Y. Mizuta, T. Shimomura and T. Sobukawa, Sobolev's inequality for Riesz potentials of functions in non-doubling Morrey spaces, Osaka J. Math. 46 (2009), no. 1, 255-271. 
[17] C. B. Morrey, Jr., On the solutions of quasi-linear elliptic partial differential equations, Trans. Amer. Math. Soc. 43 (1938), no. 1, 126-166.

[18] E. Nakai, Hardy-Littlewood maximal operator, singular integral operators and the Riesz potentials on generalized Morrey spaces, Math. Nachr. 166 (1994), 95-103.

[19] F. Nazarov, S. Treil and A. Volberg, Cauchy integral and Calderón-Zygmund operators on nonhomogeneous spaces, Internat. Math. Res. Notices 1997, no. 15, 703-726.

[20] _ Weak type estimates and Cotlar inequalities for Calderón-Zygmund operators on nonhomogeneous spaces, Internat. Math. Res. Notices 1998, no. 9, 463-487.

[21] T. Ohno and T. Shimomura, Sobolev inequalities for Riesz potentials of functions in $L^{p(\cdot)}$ over nondoubling measure spaces, Bull. Aust. Math. Soc. 93 (2016), no. 1, $128-136$.

[22] J. Peetre, On the theory of $\mathscr{L}_{p, \lambda}$ spaces, J. Functional Analysis 4 (1969), 71-87.

[23] N. G. Samko, S. G. Samko and B. G. Vakulov, Weighted Sobolev theorem in Lebesgue spaces with variable exponent, J. Math. Anal. Appl. 335 (2007), no. 1, 560-583.

[24] Y. Sawano, Sharp estimates of the modified Hardy-Littlewood maximal operator on the nonhomogeneous space via covering lemmas, Hokkaido Math. J. 34 (2005), no. 2, $435-458$.

[25] Y. Sawano, M. Shigematsu and T. Shimomura, Generalized Riesz potentials of functions in Morrey spaces $L^{(1, \varphi ; \kappa)}(G)$ over non-doubling measure spaces, Forum Math. 32 (2020), no. 2, 339-359.

[26] Y. Sawano and T. Shimomura, Sobolev embeddings for Riesz potentials of functions in non-doubling Morrey spaces of variable exponents, Collect. Math. 64 (2013), no. 3, $313-350$.

[27] K. Stempak, Examples of metric measure spaces related to modified Hardy-Littlewood maximal operators, Ann. Acad. Sci. Fenn. Math. 41 (2016), no. 1, 313-314.

[28] J.-O. Strömberg, Weak type $L^{1}$ estimates for maximal functions on noncompact symmetric spaces, Ann. of Math. (2) 114 (1981), no. 1, 115-126.

[29] Y. Terasawa, Outer measures and weak type $(1,1)$ estimates of Hardy-Littlewood maximal operators, J. Inequal. Appl. 2006, Art. ID 15063, 13 pp. 


\section{Takao Ohno}

Faculty of Education, Oita University, Dannoharu Oita-city 870-1192, Japan

E-mail address: t-ohno@oita-u.ac.jp

\section{Tetsu Shimomura}

Department of Mathematics, Graduate School of Humanities and Social Sciences, Hiroshima University, Higashi-Hiroshima 739-8524, Japan

E-mail address: tshimo@hiroshima-u.ac.jp 